\title{
Non-functioning pituitary adenomas, not all the same and certainly not boring!
}

\author{
Maria Fleseriu ${ }^{1,2}$ (D) Niki Karavitaki ${ }^{3,4}$
}

Published online: 13 February 2018

(c) Springer Science+Business Media, LLC, part of Springer Nature 2018

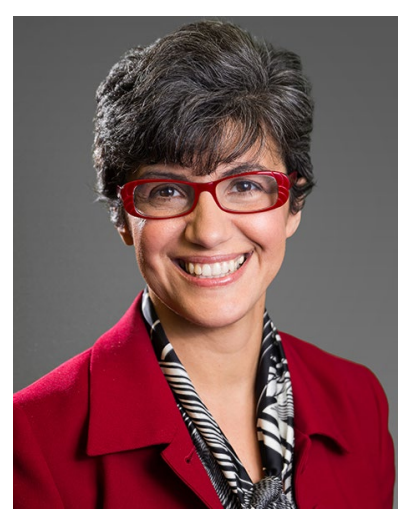

Non-functioning pituitary adenomas (NFAs) or, as recently proposed, "non-functioning pituitary neuroendocrine tumors" are benign neoplasms that arise from the adenohypophyseal cells and are not associated with clinical evidence of hormonal hypersecretion. As lack of clinical manifestations of hormonal hypersecretion frequently results in significant diagnostic delay, they are usually detected when they are large enough to induce mass effects to surrounding structures. When treatment is indicated, surgery is the first option, combined or not with adjuvant radiotherapy and/or medical therapy (off-label for NFAs); nonetheless, an optimal followup algorithm is still unclear. Furthermore, with increased accessibility to high-quality imaging, more asymptomatic NFAs are diagnosed every year adding further burden on the

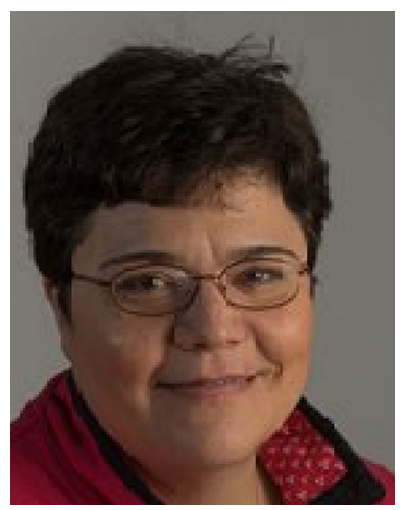

Maria Fleseriu

fleseriu@ohsu.edu

1 Department of Medicine, Division of Endocrinology, Diabetes \& Clinical Nutrition, Portland, OR, USA

2 Department of Neurological Surgery, and Northwest Pituitary Center, Oregon Health \& Science University, Portland, OR, USA

3 Institute of Metabolism and Systems Research, College of Medical and Dental Sciences, University of Birmingham, Birmingham B15 2TT, UK

4 Centre for Endocrinology, Diabetes and Metabolism, Birmingham Health Partners, Birmingham B15 2TH, UK health care system and anxiety to patients. The fourth edition of the World Health Organization Classification of Tumors of the Pituitary Gland adopts a classification based on cell lineage and in addition to hormone immunohistochemistry recognizes the value of other immunohistochemical markers including transcription factors. Not all NFAs have the same clinical course and robust markers predicting their future behavior and potential aggressiveness are still under discussion. Furthermore, the role of the new WHO classification in clinical practice needs to be validated. Finally, although data is scarce, long-term studies of patients with NFA suggest that mortality higher than that of the general population pointing highlighting the need for improved management strategies.

Thus, this Pituitary special issue appears at an optimal time to gather current knowledge and understanding of NFAs, to highlight areas requiring further attention and to provide future perspectives in the field.

Ntali and Wass provide an overview on epidemiology, clinical presentation and diagnosis. These tumors account for $14-54 \%$ of pituitary adenomas with a prevalence of 7-41.3/100,000 population. Manojlovic-Gacic, Engström and Casar-Borota offer an update based on the new WHO classification of non-functioning pituitary adenomas, 
introduce the term "non-functioning pituitary neuroendocrine tumor" and give an updated overview of prognostic biomarkers. Zatelli reviews the pathogenesis of non-functioning pituitary adenomas, an enigmatic and a complex process including molecular to genetic and epigenetic modifications, tumor suppressor gene, oncogene, and cell cycle derangements. Wildemberg, Glezer, Bronstein and Gadelha detail precipitating factors for apoplexy, management options and outcomes. Management approach of NFAs depends on clinical and radiological features at diagnosis and includes surgery, radiation therapy, observation and medical treatment. Penn, Burke and Laws illustrate indications for surgery and detail the multidisciplinary and complex perioperative management of these patients. Furthermore, the authors emphasize that expertise and "excellence in pituitary surgery" requires specialized training, residency and fellowship at centers with a high volume of pituitary cases, but also performing a high number of pituitary tumor surgery annually. Minitti, Flickinger, Tolu and Paolini discuss radiotherapy options, efficacy and short- and long-term complications and define the place of this modality in management. Huang and Molitch discuss how in the absence of hypopituitarism or visual field defects, patients with NFAs may be followed by periodic brain magnetic resonance imaging surveillance. Indications for surgery in patients initially under observation are also reviewed. Even-Zohar and Greenman review data on dopamine agonists and other off-label medical treatments in preventing tumor recurrence.

The importance of morphologic classification and identification of subgroups carrying higher risk of NFA regrowth are enunciated by Raverot, Vasiljevic and Jouanneau. BenShlomo and Cooper review epidemiology, molecular pathology and clinical behavior of silent corticotroph tumors, and highlight the need for rigorous monitoring for both recurrences and hypopituitarism. Langlois, Woltjer, Cetas and Fleseriu detail epidemiology and clinical characteristics of silent somatotroph adenomas and suggest individualized follow-up for tumor recurrence and the potential of phenotype change to clinical acromegaly. Mortality in patients with non-functioning pituitary adenoma is higher compared with that of the general population. Tampourlou, Fountas, Ntali, Karavitaki review available studies and discuss factors associated with increased mortality as older age at diagnosis and female sex. Interestingly, re-growth of the NFA, presentation with acute apoplexy, cavernous sinus invasion, extent of adenoma removal and repeat surgery for relapse are not predictors of mortality. Andela, Lobatto, Pereira, Furth and Biermasz provide a literature overview of health outcomes in patients with NFA, using a conceptual health related-quality of life (QoL) model. The authors emphasized that a concrete conceptualization of health outcomes in patients with NFA can be helpful to improve clinical care and optimize QoL. Lenders and McCormack discuss the very rare pituitary carcinomas in NFAs. Factors identified as markers of the potential for aggressive behaviour, include rapid tumour growth, growth after radiotherapy, gain or shift of hormone secretion and raised proliferative markers. Temozolomide, targeted therapies or immunotherapy can be used for these challenging cases.

We are grateful to our eminent colleagues all over the world, leading international experts who generously shared their experience with us on these focused articles. We also thank the many patients who have participated in clinical trials over the years and helped advance discoveries in pituitary research and improvement in clinical practice.

Our sincere thanks to Shlomo Melmed, Pituitary Editorin-Chief, all participating authors, and the helpful Springer editorial staff for enabling a scholarly and timely issue.

We very much hope that this special issue will be a valuable tool for those involved in care of patients with NFA and will provide stimuli for further advances on our under-

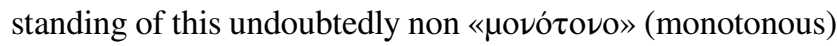
tumor type!
Guest editors

Maria Fleseriu, MD, FACE

Niki Karavitaki, MSc, PhD, FRCP 